\title{
Lifetime Maximization of Wireless Sensor Networks for a Fault Diagnosis System Using LEACH Protocol
}

\author{
Indhu RAMALINGAM, R. A. SANKARAN
}

\begin{abstract}
The breakdown in a machine created by a fault will greatly affect the plant operation. The frame work of fault diagnosis of machines using machine learning techniques is an established area. Here, the fault diagnosis system is implemented with the help of wireless sensor networks (WSN). Each machine in the plant is fitted with sensors (node) from which the fault diagnosis is carried out. The signals/messages from each node are transmitted to a base station, which acts as a central control unit for the entire plant. Fault diagnosis using WSN in a factory setup has few challenges. The major issue in WSN is the life time of the nodes, as they are situated far away from base station in many plants like thermal power plant, refinery, and petroleum industries. To address the life time of the nodes many researchers have developed many protocols like LEACH, SEP, ERP, HCR, HEED, and PEGASIS. The plants need customisation in terms of choosing suitable algorithms and choosing location of the base station within the plant for better life time of the nodes. This paper presents results of both experimental and simulation studies of a typical plant, where the vibration signals from each machine are acquired and through machine learning techniques the fault diagnosis is performed with the help of wireless sensor networks. For illustrative purpose, a well reported bearing fault diagnosis data set is taken up and fault diagnosis case study was performed from wireless sensor networks point of view (experimental study). Here, at every stage, the computational time is taken as a primary concern which affects the life time of the sensor nodes. Then, the WSN of 18 sensor nodes representing 18 machines with LEACH protocol is simulated in Matlab@ to study the life time characteristics of each node while keeping base station at different locations. The life time of different nodes is heavily dependent on the location of the base station. Finding the right location of the base station for a given plant is another contribution of this work.
\end{abstract}

Keywords: fault diagnosis; life time maximization; machine learning; wireless sensor networks

\section{INTRODUCTION}

In a plant, there are a number of machines whose health status is critical and responsible for the plant shut down. In order to minimize the shut down time and maximize the utilisation of the plant, many predictive maintenance strategies were proposed, developed and implemented. Out of them, machine learning based fault diagnosis (predictive method) is most recent one. The data (signals) acquired for the purpose of fault diagnosis were transmitted to a central control system through wires/cables. These cables are often disturbed by other plant activities. Also, there is a possibility of collecting noise, especially, when signals are taken near to high power electric lines. To handle these issues, industrialist started using wireless sensor networks. Implementation of the fault diagnosis system through wireless sensor networks solved the wire/cable related issues; however, it posed a new set of problems/challenges [1-2]. For each machine one sensor unit with a radio for transmitting the data is fixed. Normally, these sensor nodes are operated by batteries. As many types of machinery in the plants are generally operated by very high electric power, and continuous power supply is needed for WSNs, industrialists often preferred a battery operated sensor nodes. The batteries in the sensor nodes need frequent replacement if the WSN is not designed properly.

In vibration based fault diagnosis systems, a large amount of data needs to be transmitted to base station (central control unit) for processing and decision making. This results in wastage of battery energy and memory space of the sensor nodes, thereby increasing the cost of maintenance and network traffic/collisions and reducing the reliability of the data communication process. The memory related issues are discussed by Bao et al., while dealing with accelerometer signals in structural health monitoring application [3]. However the scope of the study is to increase the sensor node lifetime mainly by improving the data processing techniques and base station location. There are many approaches for lifetime maximization like power saving methods [4], decentralized data processing [5] and data compression techniques [6]. Each one of them has its own pros and cons in terms of throughput, reliability of data received, speed transmission and cost associated with it.

The proposed system describes that the wireless solution works at a high refresh rate at low cost. Commercial-Off-The-Shelf (COTS) hardware (XBee module from Maxstream) was also used for this solution. Plastic fabrication is done for a real time industrial application with 4 temperature nodes having a cycle time of $128 \mathrm{~ms}$. The experimental outputs show that the node lifetime of the above 4 nodes is 4 Months. As the temperature varies very slowly the sampling and payload data transmission rates of the above experiment are around $8 \mathrm{~Hz}$ and are considered less [7].

The proposed system in [8] uses both wired and wireless communication approaches for SCADA (Supervisory control and data acquisition). Small microprocessors are used in ISM's (Intelligent Sensor Module) and Gateways. Large microprocessors are used in RDAU (Remote Data Acquisition Units). In ISM, the sampling rate of up to 11 samples per cycle maximum of $660 \mathrm{~Hz}$ is found and it can be used to analyse till fifth harmonic of the signal. IN RDAU, the sampling rate is 68 samples per cycle which can be used to analyse up to 34 harmonic of the signal. The next level of this is a Dynamic Power Management (DPM) protocol used for a WSN, which can consume less battery; hence the node lifetime is increased by $33 \%$.

The system in [9] is proposed for machinery condition-based maintenance which uses commercial WSN products. Single Hop topology is used for shortrange transmissions, latency requirements, to simplify the nodes and for less physical space for machineries. The requirements for the system are four sensor nodes, a base 
station, and a computer with LabView7.1 for GUI and for processing the signals. A heat generating plant and airconditioning plant can be installed with the proposed system. The data processing is not done at the sensor nodes, as it will consume more energy, still the energy consumption was very high and it was around $2500 \mathrm{mAh}$ for 9 days if each sensor node sends data within 2 seconds.

In [10] WSN, online Remote Energy Monitoring and fault diagnosis for industrial applications is proposed. The inputs for the sensor nodes present in the system are Motor terminal data, two line-to-line voltages, two phase current, and a shaft torque, then the nodes transmit the received data and it is finally processed in the Central Supervisory Station (CSS). Rotor eccentricity fault was found using Simulation. The feasibility of the proposed system for fault diagnosis is found using FFT on the single phase current signal. The IEEE 802.15.4 compliant CC2420 radio components are used in the sensor nodes. ZigBee/IEEE802.15.4 based WSN for an Induction motor health monitoring system which has imbalance fault was proposed. The motor vibration signature was measured using tri-axial accelerometer ADXL330. CC2430, an IEEE802.15.4 standard compliant transceiver from Texas Instruments was used for developing a sensor node. The sensor node just communicates to the base station, all other processing was done in the base station using MATLAB. The proposed system has different levels of rotor imbalance faults created in the induction motor to find the results.

A wireless vibration monitoring system (WiMon) is about WirelessHART proposed and developed by ABB [11]. The number of sensor units present in the system is 100. It also has Gateway, WiMon Data Manger, Object Linking and Embedding for Process Control server, ABB Analyst, vibration sensor, temperature sensor, battery and WirelessHART radio. The proposed system supports IEEE 802.15.4 radio and WirelessHART network standards. If the waveform was transmitted once a day and vibration root mean square (RMS) and temperature values were given once per hour, the lifetime of the battery was around 5 Years.

The proposed system [12] has an essential insight mesh to monitor the conditions as a wireless solution. It is proposed by GE which had wSIM (Wireless mesh network node), wSIM Repeater, Manager Gateway and transducers. IEEE 802.15.4 compliant radio and wireless mesh communications was used and IEEE 802.15.4 was the gateway for the communication interfaces. WSIM sensor node supports maximum of 4 temperature or vibration transducers. The sample interval was minimum 15 minutes for static vibrations and 24 hours for dynamic vibrations. The battery lifetime was 3 years with standard configurations if the static data was sent every 2 hours from all channels and dynamic data was sent once per day from all channels.

In WSN, a typical battery capacity might be $10.8 \mathrm{~kJ}$ at $3 \mathrm{~V}$ [13]. The power consumed is about 25 to $100 \mathrm{~mW}$ during transmission or reception. This gives the sensor node the life time of about four months. This lifetime depends on the amount of data transmitted and how frequently the data are transmitted. In wired sensor networks, huge amount of data are handled for fault diagnosis purpose which wireless sensor networks systems cannot afford to manage due to limited battery power available. Hence, there is a need to reduce the power consumption in every stage of fault diagnosis process like feature extraction, feature selection, classification, finding the shortest path to base station etc.

In order to illustrate the method, an experimental case study of bearing fault diagnosis is taken up. There are 18 machines considered in a plant, each having a sensor radio node for transmitting the data. There is one base station (central control unit), which will collect data from all 18 nodes and present it to the maintenance Engineers. Upon going through the literature, one can find that in machine learning based fault diagnosis, many features like statistical features [14], Histogram features [15], ARMA features [16], wavelet-DWT features [17], and classifiers (18) were used. In most of the study on fault diagnosis using machine learning approach, the focus was on achieving high classification accuracy so that when a fault occurs, it can be identified successfully. However, a small sacrifice in classification accuracy may give computationally effective features and classifiers. Computationally effective techniques use relatively less battery power. Hence, the first part of the study is to find the best feature-classifier combination for fault diagnosis of bearings using machine learning approach. At every stage, the decision is made from wireless sensor networks point of view to save battery energy. Here, along with good condition of the bearings, three fault conditions (inner race fault, outer race fault, inner and out race fault) are also considered [19]. With this set up, one can build the best possible wireless sensor network in an industrial environment.

The next step is to reduce power consumption in transmitting the data to base station. There are a number of protocols available for energy efficient hopping like HCR [20], HEED [21], PEGASIS [22], ERP [23], SEP [24] and LEACH [25]. In all these WSNs, the location of the base station plays a critical role in lifetime of individual nodes in the network. One has to study in detail to understand the energy dissipation behaviour of nodes for different configuration of the networks. Hence, the second part of the study is to simulate the plant with 18 sensor nodes in which LEACH protocol is implemented. The location of the base station is varied and WSN performance was studied to find the best suited location for the base station from energy perspective.

To sum up, the contribution of the paper is twofold in WSN based fault diagnosis system of a plant:

(i) Optimal design of feature-classifier combination from WSN perspective for fault diagnosis of machine component using vibration signals.

(ii) Optimal location of the base station in WSN set up for LEACH protocol using simulation studies.

\section{EXPERIMENTAL SETUP}

The general architecture used for the fault diagnosis system uses wireless sensor network which has one base station and a number of sensor nodes as shown in Figure 1. The radio present in the sensor node uses IEEE 802.15.4 and ZigBee protocols for transmission of vibration [13]. 


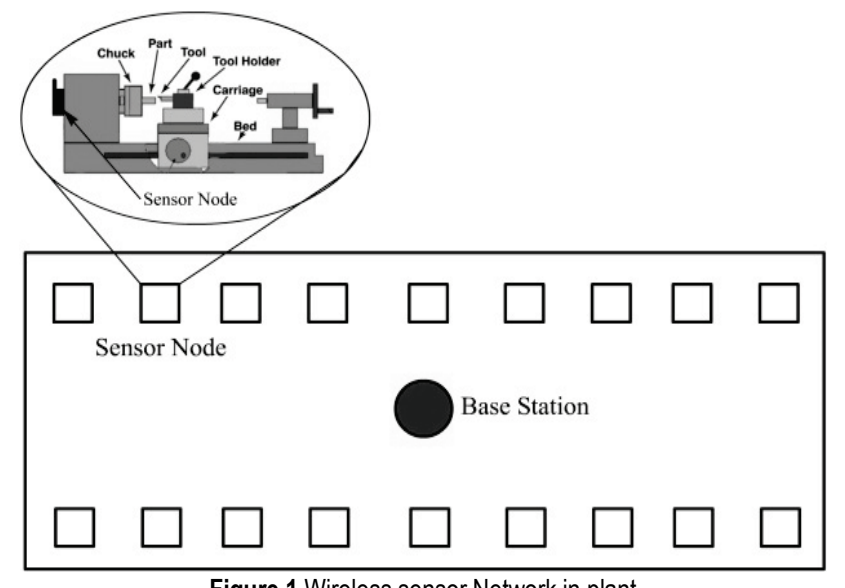

Figure 1 Wireless sensor Network in plant

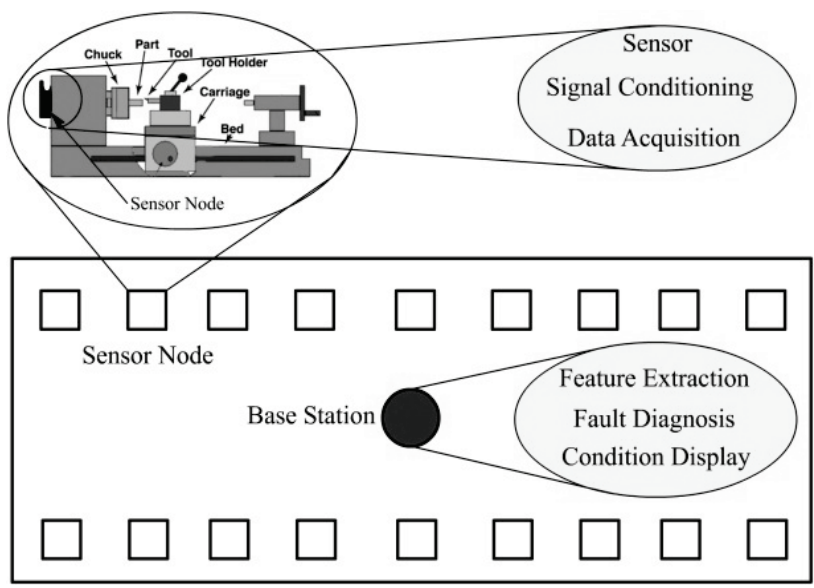

Figure 2 WSN with signal conditioning and data acquisition

Fig. 1 illustrates the working principle of the proposed system. Each sensor node was used for the measurement of the machine vibration signature. The fault diagnosis process consists of five stages namely, signal conditioning, data acquisition, feature extraction, feature classification and display of the condition. In the present study, piezo-electric transducers (Dytran make) were used, which have in-built signal conditioning circuits (pre-amplification). It was mounted on the flat surface using direct adhesive mounting technique. The accelerometer was connected to the signal-conditioning unit (DACTRAN FFT analyser), where the signal goes through the charge amplifier and an Analogue-to-Digital Converter (ADC). The acquired signals were stored in the node's memory [26]. To illustrate the process, bearing faults like inner race fault, outer race fault and inner and outer race fault were created and the corresponding vibration signatures were taken. The footstep in [13] is followed to process the acquired vibration signals.

In our case in Fig. 2, the signal conditioning and data acquisition was done at the sensor nodes. The sensor nodes were used like a wireless DAQ (data acquisition system).

Here, a large amount of vibration signals need to be transmitted to the base station, which increases the number of packets and in turn increases the energy dissipation from node's battery. In the light of the above, the case in hand was studied through simulation and energy consumption patterns are reported in detail in the results and discussion section.

\section{DESIGN OF FAULT DIAGNOSIS SYSTEM}

In the present study, machine learning approach was used for fault diagnosis. Machine learning has three distinct phases, namely, feature extraction, feature selection and classification. Effective design of fault diagnosis system involves choosing appropriate items at every phase of machine learning with less computational time. Minimum computational time will minimize the energy dissipated by the processor in the nodes in wireless sensor networks. The commonly used features are statistical features, histogram features, Autoregressive moving average (ARMA) features, and wavelet features. In this study, all four features were used and suitable feature was selected for WSN based systems. Even at feature selection stage, minimal number of features was selected to reduce the computational load at sensor node to save some battery power. There were 26 classifiers used for classifying the condition and the best classifier was chosen which gives high classification accuracy with minimal computation time.

\subsection{Choosing Features}

Table 1 Features present in the tree

\begin{tabular}{|c|l|c|c|c|}
\hline S. No. & Statistical Features & $\begin{array}{c}\text { Histogram } \\
\text { Features }\end{array}$ & $\begin{array}{c}\text { ARMA } \\
\text { Features }\end{array}$ & $\begin{array}{c}\text { Wavelet } \\
\text { Features }\end{array}$ \\
\hline 1 & Kurtosis & H3 & A1 & V3 \\
\hline 2 & Standard Error & H1 & K3 & V4 \\
\hline 3 & Range & H2 & A3 & V1 \\
\hline 4 & Minimum & H4 & K1 & V13 \\
\hline 5 & Median & H8 & E1 & - \\
\hline 6 & Skewness & - & - & - \\
\hline 7 & Mean & - & - & - \\
\hline
\end{tabular}

Table 2 Feature selection based on time accuracy

\begin{tabular}{|c|c|c|c|c|c|c|c|c|}
\hline \multirow{2}{*}{$\begin{array}{c}\text { Number of } \\
\text { Features }\end{array}$} & \multicolumn{2}{|c|}{ Statistical Features } & \multicolumn{2}{|c|}{ Histogram Features } & \multicolumn{2}{|c|}{ ARMA Features } & \multicolumn{2}{|c|}{ Wavelet Features } \\
\hline & $\mathrm{CA}(\%)$ & Time (s) & $\mathrm{CA}(\%)$ & Time (s) & $\mathrm{CA}(\%)$ & Time (s) & $\mathrm{CA}(\%)$ & Time (s) \\
\hline First 7 & 90.50 & 0.02 & - & - & & & & \\
\hline First 6 & 91.00 & 0.01 & - & - & & & & \\
\hline First 5 & 91.25 & 0.01 & 90.25 & 0.02 & 95.75 & 0.02 & & \\
\hline First 4 & 92.00 & 0.01 & 90.50 & 0.02 & 95.50 & 0.01 & 97.25 & 0.01 \\
\hline First 3 & 90.75 & $0.00 *$ & 89.25 & 0.02 & 95.50 & 0.01 & 96.75 & 0.01 \\
\hline First 2 & 91.00 & 0.02 & 90.25 & 0.01 & 95.50 & 0.01 & 96.50 & 0.01 \\
\hline First 1 & 87.25 & $0.00^{*}$ & 73.00 & 0.01 & 77.50 & 0.01 & 94.25 & 0.01 \\
\hline
\end{tabular}

\subsection{Choosing Classifier}

Referring to Tab. 2, the highest classification accuracy is obtained with wavelet features and multiclass classifier. This was arrived at after classifying the selected 
features from each type (as shown in Tab. 1) with 26 different classifiers. Here, the computation time is a little high. For efficient sensor node power management, not only high classification accuracy but also low computation time is needed. Hence, Referring to Tab. 2, IBK and Kstar classifiers were chosen as the best classifiers.

Table 3 Classification based on time accuracy

\begin{tabular}{|c|c|c|c|c|c|c|c|c|c|}
\hline \multirow{2}{*}{ S. No } & \multirow{2}{*}{ Classifier } & \multicolumn{2}{|c|}{ Statistical Features } & \multicolumn{2}{|c|}{ Histogram Features } & \multicolumn{2}{|c|}{ ARMA Features } & \multicolumn{2}{|c|}{ Wavelet Features } \\
\hline & & $\mathrm{CA}(\%)$ & Time (s) & $\mathrm{CA}(\%)$ & Time (s) & $\mathrm{CA}(\%)$ & Time (s) & $\mathrm{CA}(\%)$ & Time (s) \\
\hline 1 & Bayesnet & 89.50 & 0.01 & 88.50 & 0.02 & 94.50 & 0.02 & 94.25 & 0.02 \\
\hline 2 & NaiveBayes & 88.25 & 0.00 & 86.25 & 0.02 & 93.00 & 0.01 & 95.50 & 0.01 \\
\hline 3 & NaiveBayesUpdateable & 88.25 & 0.00 & 86.25 & 0.00 & 93.00 & 0.01 & 95.50 & 0.00 \\
\hline 4 & Logistic & 90.25 & 0.09 & 88.75 & 0.05 & 93.50 & 0.04 & 97.75 & 0.17 \\
\hline 5 & MultiLayerPerception & 89.50 & 0.67 & 89.25 & 0.49 & 93.75 & 0.39 & 97.50 & 0.73 \\
\hline 6 & Simple Logistic & 90.25 & 0.25 & 89.00 & 0.18 & 93.25 & 0.16 & 97.25 & 0.02 \\
\hline 7 & Ibk & 86.75 & 0.00 & 86.00 & 0.01 & 91.50 & 0.01 & 98.25 & 0.01 \\
\hline 8 & Kstar & 91.00 & 0.00 & 88.50 & 0.01 & 92.75 & 0.01 & 98.25 & 0.01 \\
\hline 9 & LWL & 89.25 & 0.00 & 90.25 & 0.01 & 87.25 & 0.01 & 91.75 & 0.00 \\
\hline 10 & AttributeSelectedClassifier & 90.25 & 0.01 & 90.25 & 0.02 & 94.25 & 0.01 & 96.25 & 0.01 \\
\hline 11 & Bagging & 91.25 & 0.02 & 86.75 & 0.02 & 94.25 & 0.01 & 96.00 & 0.02 \\
\hline 12 & ClassificationViaRegression & 90.25 & 0.06 & 89.50 & 0.06 & 94.50 & 0.05 & 97.25 & 0.05 \\
\hline 13 & IterativeClassifierOptimizer & 90.50 & 0.34 & 87.25 & 0.27 & 94.25 & 0.20 & 97.25 & 0.37 \\
\hline 14 & LogitBoost & 91.00 & 0.05 & 87.50 & 0.03 & 94.00 & 0.02 & 97.00 & 0.05 \\
\hline 15 & MultiClassClassifier & 89.75 & 0.05 & 89.00 & 0.05 & 92.00 & 0.02 & 98.50 & 0.11 \\
\hline 16 & RandomCommitee & 88.75 & 0.02 & 84.50 & 0.01 & 91.50 & 0.01 & 97.25 & 0.02 \\
\hline 17 & RandomSubSpace & 90.00 & 0.02 & 87.25 & 0.02 & 94.50 & 0.01 & 96.00 & 0.02 \\
\hline 18 & DecisionTable & 89.00 & 0.02 & 87.75 & 0.02 & 94.50 & 0.01 & 94.75 & 0.03 \\
\hline 19 & Jrip & 88.25 & 0.03 & 88.50 & 0.02 & 93.75 & 0.02 & 96.00 & 0.01 \\
\hline 20 & Part & 91.75 & 0.01 & 90.50 & 0.01 & 94.25 & 0.01 & 96.00 & 0.01 \\
\hline 21 & HoeffdingTree & 88.50 & 0.01 & 86.25 & 0.01 & 93.25 & 0.01 & 95.25 & 0.01 \\
\hline 22 & $\mathrm{~J} 48$ & 92.00 & 0.00 & 90.75 & 0.02 & 94.25 & 0.01 & 95.75 & 0.01 \\
\hline 23 & LMT & 90.50 & 0.41 & 89.00 & 0.27 & 92.50 & 0.03 & 97.25 & 0.48 \\
\hline 24 & RandomForest & 91.00 & 0.11 & 88.00 & 0.01 & 92.25 & 0.05 & 97.25 & 0.09 \\
\hline 25 & RandomTree & 88.25 & 0.00 & 84.50 & 0.01 & 91.50 & 0.01 & 97.00 & 0.00 \\
\hline 26 & REPTree & 88.75 & 0.00 & 88.75 & 0.02 & 94.00 & 0.01 & 96.25 & 0.02 \\
\hline
\end{tabular}

\section{SIMULATION OF WIRELESS SENSOR NETWORKS}

Once the fault diagnosis system is designed, then the next step in the sensor node is choosing the radio parameters. The general architecture of the sensor node is shown in Fig. 3. As stated earlier, the sensor here is piezoelectric sensor and power unit is a battery. Any commercially available RF module can be used as radio. Generally, any microprocessor/controller with in-built memory is used in the sensor nodes. In the present study, the wireless sensor node parameters are taken as shown in Tab. 4 for the simulation study. The plant has totally 18 machines in which the sensor nodes are attached and the machines are having fixed location and hence, the sensor nodes also have fixed location.

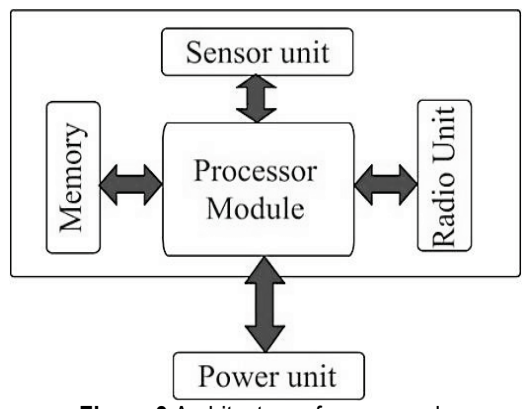

Figure 3 Architecture of sensor node

The sensor nodes acquire vibration signals from the machines (bearings - for illustration) and transmit the signals to the base station/central control unit. In the process, the energy of the battery is dissipated. The energy dissipated is affected mainly by the amount of data transmitted, the frequency of the data transmission, the distance between the node to base station and the path travelled by the data (routing). There are many studies already reported in literature for routing, scheduling as the energy dissipated is more in these activities. The distance, frequency of data transmitted and amount of data transmitted also affect the energy dissipation; however, the amount of energy saved is relatively less compared to the routing and scheduling. Thus, this area was not much investigated in detail.

Table 4 WSN simulation parameters

\begin{tabular}{|c|l|c|}
\hline S.No. & \multicolumn{1}{|c|}{ Parameter } & Value \\
\hline 1. & No. of nodes & 18 \\
\hline 2. & Plant area & $250 \times 150 \mathrm{~m}$ \\
\hline 3. & No. of base station & 1 \\
\hline 4. & Initial energy of nodes & $0.5 \mathrm{~J}$ \\
\hline 5. & Energy for transferring a bit (ETX) & $5 \times 10^{-8} \mathrm{~J}$ \\
\hline 6. & Energy for receiving a bit (ERX) & $5 \times 10^{-8} \mathrm{~J}$ \\
\hline 7. & Free space energy (EFS) & $1 \times 10^{-11} \mathrm{~J}$ \\
\hline 8. & Multipath Energy (MPE) & $1.3 \times 10^{-15} \mathrm{~J}$ \\
\hline 9. & Data Aggregate Energy (DAE) & $5 \times 10^{-9} \mathrm{~J}$ \\
\hline 10. & Routing Protocol & $\mathrm{LEACH}$ \\
\hline 11. & Control Packet Length (Bytes) & 200 \\
\hline 12. & Packet length (Bytes) & 4500 \\
\hline
\end{tabular}


This paper uses LEACH protocol for illustration and focuses on the energy dissipation of the sensor nodes for the following cases:

1) Various locations of the base station within the plant

2) The sensor node transmits vibration signals directly to base station (Refer Fig. 2).

\subsection{Location of Base Station}

As mentioned earlier, the locations of sensor nodes are fixed for the given plant layout. The challenge is to find the location of the base station. The objective here is to have maximum 'rounds' before any sensor node becomes dead. The advantage in wireless sensor networks is that even if one node becomes dead, the routing algorithm finds the alternate path to reach the base station. Therefore, it is possible to keep the WSN working, in spite of some dead nodes. However, if the number of dead nodes is higher, then from application point of view, it is not acceptable. Hence, one has to find a threshold value for maximum number of dead nodes that is acceptable for the plant standards. In the present study, it is selected as 9 , not only due to $50 \%$ of the total nodes, but also beyond this point the rounds are not linearly proportional to the number of packets transmitted. However, for each 'number of dead nodes', the simulation was performed to find the 'rounds' as a measure of amount of data transmitted. A study was carried out to find the best location for the base station.

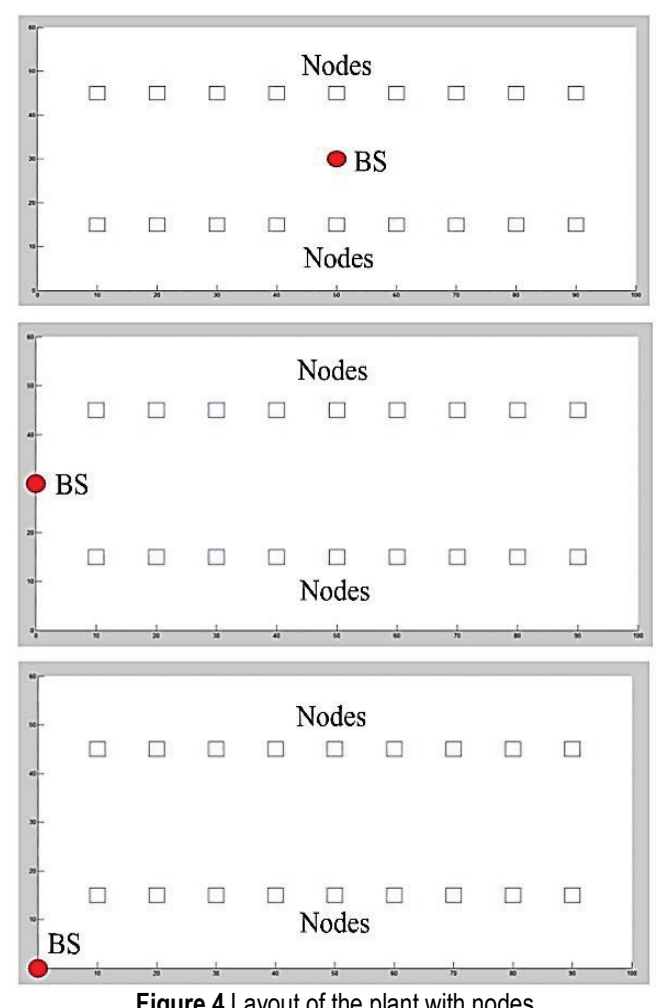

Figure 4 Layout of the plant with nodes

First, the possible locations were identified like centre point of the plant, corners of the plant, middle extreme points as shown in Fig. 4. Since the plant considered in the case study is symmetric, it is enough to conduct study in a quarter model and the results can be applied to the full model of the plant. With 18 nodes the LEACH protocol simulation was performed till all the 18 nodes died.

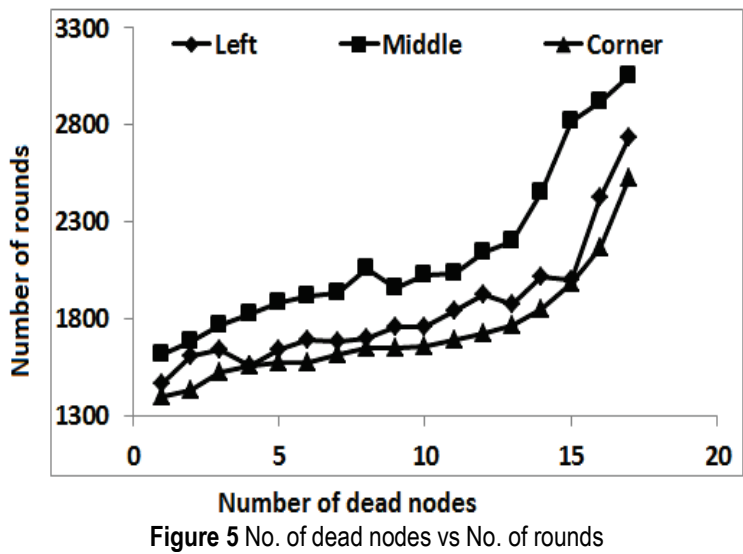

The results are presented in Fig. 4. The number of rounds represent the lifetime of the nodes indirectly. Referring to Fig. 4, one can observe that when base station is in centre, the network is having more life (rounds) with nine dead nodes option. Even, for other number of dead nodes, the maximum life for base station is achieved by the centre position. Hence, it becomes an obvious choice for the base station location.

When the number of dead nodes is 18 , the rounds are reaching 10000 , which is a maximum round set for the simulation. This indicates that the 18th node is not dying even at 10000 rounds. The experiment could have been repeated with more rounds to check the life of the 18th node; however, it is a trivial case from industrial application point of view. Hence, it is left as it is. As per industry standards, if 17 nodes are dead, they will not keep just one node and run the industry. In reality, even if two or more nodes are dead, they will replace the battery. To study the energy dissipation pattern, this study was carried out.

\subsection{Amount of Data Transmitted}

Once the base station is fixed, the energy transmitted is affected by the amount of data transmitted. To study the effect of amount of data transmitted on the energy dissipated by the battery, a set of simulation studies were carried out with the WSN parameters given in Tab. 4. Till 9 nodes die, the study was conducted and results are presented in Fig. 6. One can observe from Fig. 6 that it takes about 1200 rounds for the first round to die. Due to hopping, the nodes choose different path to reach base station as per LEACH algorithm. For 9 nodes to die, it takes about 1960 rounds and till that time energy is dissipated linearly with rounds. The number of packets transmitted to base station is also proportional to the rounds. Beyond this point, they are not linearly proportional (not shown in Fig. 6).

\section{CONCLUSION}

This paper presented the results of both experimental and simulation studies of a typical plant, where the vibration signals from each machine are acquired and through machine learning techniques the fault diagnosis is 
performed with the help of wireless sensor networks. In each stage of the machine learning process, maximising the lifetime of the node was kept as one of the objectives. The WSN of 18 sensor nodes representing 18 machines with LEACH protocol is simulated in Matlab (C) to study the life time characteristics of each node while keeping base station at different locations. The location of the base station was found and the amount of data transmitted versus maximum lifetime of the nodes was studied and reported. For Industrial people, the above results will be very much useful as the framework is laid for optimising WSN for a given plant layout.
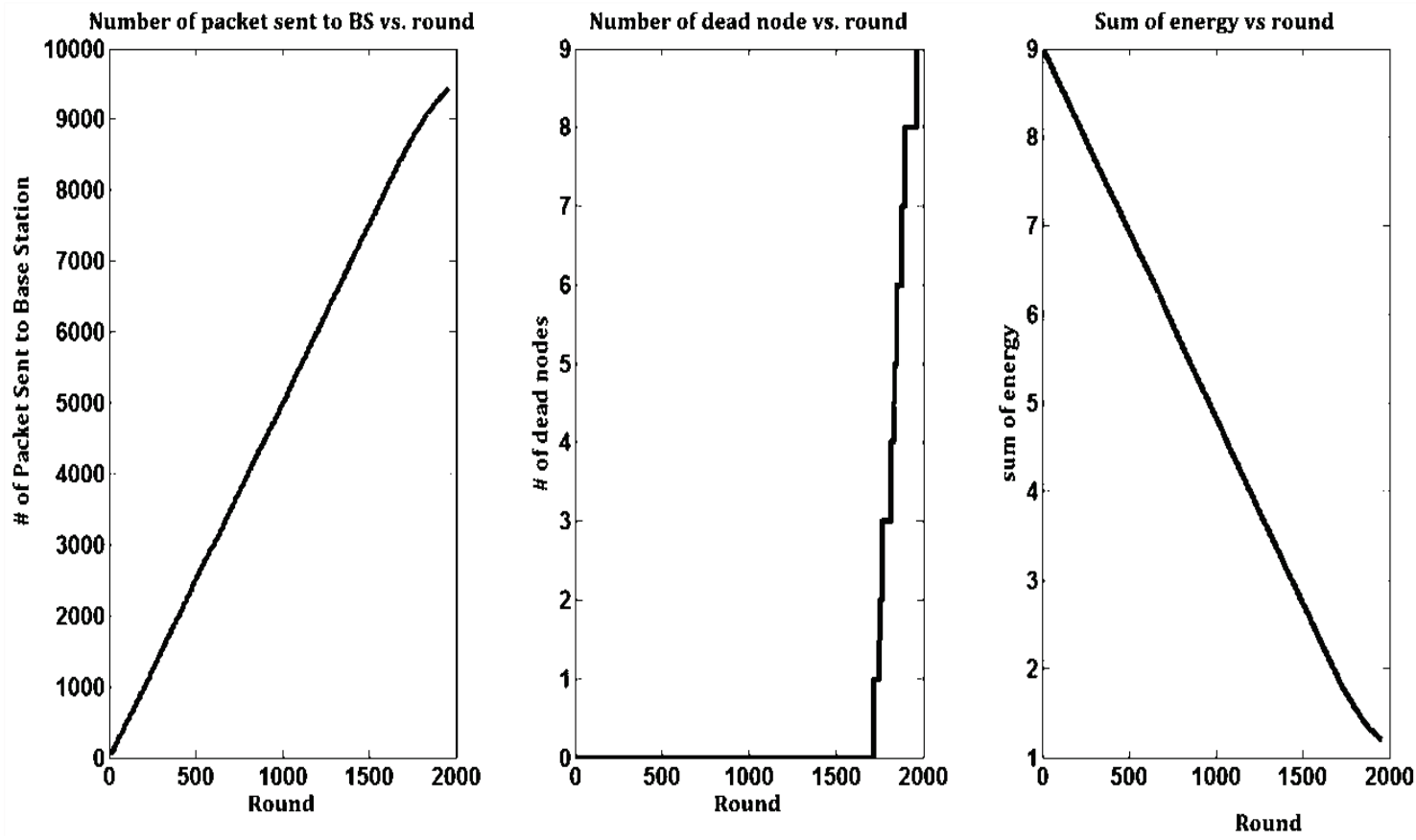

Figure 6 Sensor node transmits vibration signals directly

\section{REFERENCES}

[1] Antoniou, M., Boon, M. C., Green, P. N., Green, P. R., York, T. A. (2009). Wireless Sensor Networks for Industrial Processes. IEEE Sensors Applications Symposiums, February, 2009. https://doi.org/10.1109/SAS.2009.4801768

[2] Song, J., Mok, A. K., Chen, D., \& Nixon, M. (2006). Challenges of wireless control in pro-cess industry. Workshop on Research Directions for Security and Networking in Critical Real-Time and Embedded Systems.

[3] Bao, Y., Beck, J. L., \& Li, H. (2011). Compressive sampling for accelerometer signals in structural health monitoring. Structural Health Monitoring.

[4] Kottapalli, V. A., Kiremidjian, A. S., Lynch, J. P., Carryer, E., Kenny, T. W., Law, K. H., \& Lei, Y. (2003). Two-tierd wireless sensor network architecture for structural health monitoring, Smart Structures and Materials : Smart Systems and Nondestructive Evaluation for Civil Infrastructures. Proc. of SPIE, 5057.

[5] Lynch, J. P., Sundararajan, A., Law, K. H., Kiremidjian, A. S., \& Carryer, E. (2004). Embedding damage detection algorithms in a wireless sensing unit for operational power efficiency. Smart Materials and Structures, 13(4), 800-810. https://doi.org/10.1088/0964-1726/13/4/018

[6] Xu, N., Rangwala, S., Kant Chintalapudi, K., Ganesan, D., Broad, A., Govindan, R., \& Estrin, D. (2004). A wireless sensor network for structural monitoring. Proceedings of the $2^{\text {nd }}$ international Conference on Embedded Networked Sensor Systems, ACM, 13-24. https://doi.org/10.1145/1031495.1031498

[7] Flammini, A., Marioli, D., Sisinni, E., \& Taroni, A. (2007). A real-time wireless sensor network for temperature monitoring. IEEE International Symposium on Industrial Electronics / Vigo, 916-1920. https://doi.org/10.1109/ISIE.2007.4374900
[8] Salvadori, F., de Campos, M., Sausen, P. S., de Camargo, R. F., Gehrke, C., Rech, C., Spohn, M. A., \& Oliveira, A. C. (2009). Monitoring in industrial systems using wireless sensor network with dynamic power management. IEEE Transactions on Instrumentation and Measurement, 58, 3104-3111. https://doi.org/10.1109/TIM.2009.2016882

[9] Bergmann, N. W. \& Hou, L.-Q. (2012). Novel Industrial Wireless Sensor Networks for Machine Condition Monitoring and Fault Diagnosis. IEEE Transactions on Instrumentation and Measurement, 61(10), 2787-2798. https://doi.org/10.1109/TIM.2012.2200817

[10] Bin, L. \& Gungor, V. C. (2009). Online and remote motor energy monitoring and fault diagnostics using wireless sensor networks. IEEE Transactions on Industrial Electronics, 56, 4651-4659. https://doi.org/10.1109/TIE.2009.2028349

[11] Condition Monitoring-Wireless Vibration Monitoring System, http://www05.abb.com/global/scot/scot267.nsf/ veritydisplay/02cdc6632d9dfc40852578180053d492/\$file/ wireless $\% 20$ vibration $\% 20$ monitoring $\% 20$ system.pdf (accessed Jul. 26, 2011)

[12] Essential Insight meshBentlyNevada Wireless Condition Monitoring System for Essential Assets, http://www.gepower.com/prod_serv/products/oc/en/ downloads/gea15019a wirelesscond r6.pdf

[13] Bergmann, N. W. \& Hou, L.-Q. (2014). Energy Efficient Machine Condition Monitoring Using Wireless Sensor Networks. International Conference on Wireless Communication and Sensor Network (WCSN), 285-290. https://doi.org/10.1109/WCSN.2014.65

[14] Sugumaran, V., Muralidharan, V., \& Ramachandran, K. I. (2007). Feature selection using Decision Tree and classification through Proximal Support Vector Machine for fault diagnostics of roller bearing. Mechanical Systems and Signal Processing 21(2), 930-942. https://doi.org/10.1016/j.ymssp.2006.05.004 
[15] Sakthivel, N. R., Indira, V., Nair, B. B., \& Sugumaran, V. (2011). Use of histogram features for decision tree-based fault diagnosis of mono-block centrifugal pump. International Journal of Granular Computing, Rough Sets and Intelligent Systems, 2(1), 23-36. https://doi.org/10.1504/IJGCRSIS.2011.041458

[16] Powar, R. U. (2015). Fault Diagnosis of Roller Bearing using Vibration Signals Through ARMA Features and Tree Family Classifier. International Journal of Engineering Research \& Technology, 04(07), 688-692.

[17] Vora, S., Gaikwad, J. A., \& Kulkarni, J. V. (2015). Fault Diagnosis of Bearing of Electric Motor Using Wavelet transform and Fault Classification Based on Support Vector Machine. Advance Research in Electrical and Electronic Engineering (AREEE), 2(5), 41-46.

[18] Shen, C., Wang, D., Kong, F., \& Tse, P. W. (2013). Fault diagnosis of rotating machinery based on the statistical parameters of wavelet packet paving and a generic support vector regressive classifier. Measurement, 46, 1551-1564. https://doi.org/10.1016/j.measurement.2012.12.011

[19] Lu, W., Jiang, W., Wu H., \& Hou, J. (2012). A fault diagnosis scheme of rolling element bearing based on nearfield acoustic holography and gray level co-occurrence matrix. Journal of Sound and Vibration, 331, 3663-3674. https://doi.org/10.1016/j.jsv.2012.03.008

[20] Sabri, A. \& Al-Shqeerat, K. (2014). Hierarchical ClusterBased Routing Protocols for Wireless Sensor Networks - A Survey. International Journal of Computer Science Issues, 11(1), No 2.

[21] Ullah, Z., Mostarda, L., Gagliardi, R., Cacciagrano, D., \& Corradini, F. (2016). A comparison of HEED based clustering algorithms - introducing ER-HEED. IEEE $30^{\text {th }}$ International Conference on Advanced Information Networking and Applications, 339-345. https://doi.org/10.1109/AINA.2016.87

[22] Li, T., Ruan, F., \& Fan, Z. (2016). An Improved PEGASIS Protocol for Wireless Sensor Network. $3^{\text {rd }}$ International Conference on Computer and Computing Science, 16-19.

[23] Ahvar, E., Ahvar, S., Lee, G. M., \& Crespi, N. (2014). An Energy-Aware Routing Protocol for Query-Based Applications in Wireless Sensor Networks. The Scientific World Journal. https://doi.org/10.1155/2014/359897

[24] Jaiswal, V. P. N. \& Garg, A. K. (2012). An Efficient Protocol for Reducing Energy Consumption in Wireless Sensor Networks. International Journal of Engineering Research and Applications (IJERA), 2(3), 530-533.

[25] Heinzelman, W. B., Chandrakasan, A. P., \& Balakrishnan, H. (2002). An Application-Specific Protocol Architecture for Wireless Micro-Sensor Networks Article. IEEE Transactions on Wireless Communications, 1(4), 660-670. https://doi.org/10.1109/TWC.2002.804190

[26] Tiwari, A., Ballal, P., \& Lewis, F. L. (2007). Energyefficient wireless sensor network design and implementation for condition-based maintenance. $A C M$ Transactions on Sensor Networks, 3(23). https://doi.org/10.1145/1210669.1210670

[27] Sugumaran, V., Sabareesh, G. R., \& Ramachandran, K. I. (2008). Fault diagnostics of roller bearing using kernel based neighbourhood score multi-class support vector machine. Expert Systems with Applications, 34(4), 30903098. https://doi.org/10.1016/j.eswa.2007.06.029

[28] Kumar Patel, R., Rakesh Thapliyal, B., \& Giri, C. V. K. (2015). Application of DWT and PDD for Bearing Fault Diagnosis Using Vibration Signal. Journal of Electrical Engineering.

[29] Li, C., Sánchez, R.-V., Zurita, G., \& Diego Cabrera, M. C. (2016). Fault Diagnosis for Rotating Machinery Using Vibration Measurement Deep Statistical Feature Learning. Sensors, 16, 895. https://doi.org/10.3390/s16060895

\section{Contact information:}

Indhu RAMALINGAM, Assistant Professor

Salem College of Engineering and Technology

$\mathrm{NH}-68$, Salem-Attur Main Road, Mettupatty, Perumapalayam, Selliamman Nagar, Salem, Tamil Nadu 636111, India

indhujj@gmail.com

Dr. R. A. SANKARAN, Principal

Salem College of Engineering and Technology

$\mathrm{NH}-68$, Salem-Attur Main Road, Mettupatty, Perumapalayam, Selliamman

Nagar, Salem, Tamil Nadu 636111, India

sankaran_ra@yahoo.com 